\title{
EFECTO DE LA APLICACIÓN DE TERAPIA CONDUCTUAL EN LA ADQUISICIÓN DE DESTREZAS BÁSICAS PARA EL APRENDIZAJE, EN TRES NIÑOS CON AUTISMO
}

\author{
Hannia Cabezas Pizarro
}

\author{
Recibido 11-VI-2003 • Aceptado 12-VIII-2003
}

\begin{abstract}
Resumen: El presente estudio muestra el efecto de la utilización de terapia conductual aplicada, en tres niños con autismo, y cuyo propósito fue el de desarrollar aquellas conductas de entrada básicas para el aprendizaje como son el permanecer sentados, el control de llanto, el establecimiento de contacto ocular y la identificación del yo, consideradas habilidades básicas que deben tener los niños para incursionar en el proceso educativo. Una vez que el niño adquiere esta gama de habilidades, estos repertorios conductuales se convierten a su vez en básicos para destrezas más complejas.

Siguiendo este modelo, se trabajó una segunda área que se denominó conducta intermedia, que requiere de las habilidades previamente establecidas, y dentro de las que se citan el seguimiento de instrucciones, la discriminación de objetos y la identificación de las partes del cuerpo, a su vez este repertorio conductual se convirtió en básico para desarrollar habilidades más complejas como el reconocimiento de colores, de modo que unas conductas se convierten en requisitos de otras, hasta llevar a término un programa de intervención educativo.

El proceso de intervención educativa, contempló una gama amplia de habilidades, sin embargo para efectos de este estudio, se seleccionó una muestra representativa de cada área.
\end{abstract}

Palabras clave: Autismo, Conducta Básica, Intervención, Conducta Intermedia, Conducta Avanzada, Educación.
Por definición el autismo es un trastorno generalizado del desarrollo que se caracteriza por

"una perturbación grave y generalizada de varias áreas del desarrollo: habilidades para la interacción social, habilidades para la comunicación o la presencia de comportamientos, intereses y actividades estereotipadas." (DSM IV) (APA, 1998, p. 69).

El trastorno autista se incluye dentro de esta definición y se caracteriza por:

"la presencia de un desarrollo marcadamente anormal o deficiente de la interacción y la comunicación sociales y un repertorio sumamente restringido de habilidades e intereses" (DSM IV, APA, 1998) p. 70.

Al carecer el niño con autismo, de los repertorios básicos de entrada que le permitan adquirir cualquier aprendizaje, es necesario que los programas educativos en sus niveles iniciales, se centren en la adquisición de estas destrezas.

En Costa Rica la integración y el derecho a Educación de la personas con necesidades educativas especiales es una realidad.

Se han dado cambios significativos en la sociedad en relación con esta población reconociéndose su educación como un derecho dentro de la legislación costarricense, por más severa que sea la discapacidad que el niño presente. Esta ley llamada Ley de igualdad de oportunidades para personas con discapacidad aprobada por la 
Asamblea Legislativa en el año 1996, o ley 7600 se refiere en su artículo № 33 a los Programas de estudio en la educación regular, en donde la

"programación educativa de los estudiantes con necesidades educativas especiales de la educación regular, regirán los mismos objetivos de los programas de estudio establecidos en todos los ciclos de Educación General Básica y en la Educación Diversificada, con las adecuaciones de acceso al currículo y curriculares que requieran." (1996) p. 68.

Debido a que el autismo afecta una gama muy amplia de las áreas de desarrollo, los sistemas de intervención deben ser planeados y llevados a término por personas competentes en las diferentes disciplinas como: Educación, Psicología, Medicina, Trabajo Social y otros campos de apoyo, ya que la participación de los profesionales en las diferentes áreas es de vital importancia para desarrollar una tarea integral y de conjunto.

La conjugación de factores como la detección temprana, el tratamiento médico, el apoyo psicológico a los padres, y la ejecución en las áreas educativas son la columna vertebral en donde se apoya la integración del niño al medio en que se desenvuelve.

Desde el punto de vista educativo, las necesidades de los niños con autismo se centran en tres áreas problemáticas: Adquisición de patrones lingüísticos, falta de relaciones sociales e inconsistencia 0 irregularidades en las reacciones conductuales. Steven (1984).

Si bien la dificultad en la adquisición de patrones lingüísticos está presente, y esto limita la comunicación, es necesario desarrollar antes de implementar programas en esta área, aquellas habilidades básicas, para esta conducta, lo que facilitará su aprendizaje.

En esta investigación participaron 3 niños con edades de 3 y 6 años. Los tres niños tenían un diagnóstico de autismo dado por un profesional en el campo, y ninguno había recibido atención especializada.
Dentro de las características más sobresalientes ninguno de los tres permanecía sentado, no establecían contacto ocular, y carecían de lenguaje comunicativo. Presentaban movimientos estereotipados y una marcada insistencia en hacer que las cosas permanecieran iguales.

Para efectos de este artículo se presentan las conductas trabajadas bajo los apartados, conducta básica, conducta intermedia, y conducta avanzada, atendiendo al orden de dificultad y a los prerrequisitos de entrada para cada una de ellas. Se expone en cada apartado solo una o dos que representen el área, y que ejemplifiquen para una mejor comprensión, del trabajo llevado a cabo, sin embargo cabe mencionar que la intervención educativa en su totalidad, abarcó un total de ciento seis habilidades. Este artículo, es parte de un estudio longitudinal cuya duración fue de 5 años, y en el que los niños fueron atendidos en sesiones dos veces por semana de una hora de duración cada una.

\section{Conducta básica}

Bajo este apartado se incorporaron aquellos repertorios que permitieron a los niños involucrarse efectivamente en el proceso de enseñanza aprendizaje. Se les llama "básicas", porque son el soporte sobre las que se planifica el resto del aprendizaje ya que si el niño carece de ellas, por sí solo, no sabe aprender.

Sin embargo, estas habilidades se convierten a su vez en básicas, para campos más complejos que requieran de conocimientos previos para ser incorporados por los niños, dentro de su repertorio, y así sucesivamente.

La terapia de los niños con autismo, debe provenir de "afuera hacia adentro", ya que el niño carece de aquellas habilidades que le permiten aprender, y no de "adentro hacia fuera" por la poca representatividad mental, característica que fue objeto de estudio por Rutter y Lockyer 
(1967). Lo que mejores resultados ha dado con esta población, ha sido el uso de modificación de conducta.

La persistencia en los comportamientos disruptivos como rabietas, agresión, tirar cosas, comúnmente presentes en los niños con autismo, interfieren notablemente con su aprendizaje y deben ser controlados antes de establecerse nuevas habilidades académicas. Se tomó como punto de partida la educación del niño y la incorporación de los comportamientos a través de la aplicación de técnicas conductuales, que le permitieran integrarse mejor al aula. Se trabajó el permanecer sentado por un período determinado de tiempo, y que se mantuvieran sin llorar como conductas de entrada. Posteriormente, el contacto ocular, ya que una de las características de los niños con este síndrome es la incapacidad para fijar su mirada en las personas que les hablan. Tinbergen (1962).

Así mismo, el DSM IV (1995) apunta que

"las deficiencias de la interacción social son importantes y duraderas. Puede darse una notoria afectación de la práctica de comportamientos no verbales múltiples" (p. ej. contacto ocular, expresión facial, posturas y gestos corporales) p. 70 .

La primera comunicación la tenemos con una persona en el momento en que vemos sus ojos. El establecimiento de este contacto ocular con las personas, con los objetos, con las cosas, son necesarias en el proceso enseñanza aprendizaje, de tal manera que se convierte en una conducta básica para que sobre ella se desarrollen repertorios cada vez más complejos.

Rutter y Lockyer (1967) señalaron que dentro de las características específicas que acompañan a los niños con autismo, se encuentra la poca representatividad mental, la repetición de palabras y el uso indebido de pronombres. Como parte de ello, los niños con autismo, hablan en tercera persona, sin tener una identificación de su propio yo.

La identificación del cuerpo, y la incorporación del concepto de "yo", son el punto de partida para la aprehensión de los conocimientos. A través del cuerpo, es que el niño establece la relación entre él y las personas, él y los objetos, él y las cosas que le rodean.

$$
\text { Vayer, (1977), enfatiza en que }
$$

"la personalidad se elabora progresivamente a través de la acción del yo frente al mundo exterior. Estas constantes relaciones e interrelaciones yo-mundo de las cosas, mundo de los demás, nos explican la complejidad de la experiencia corporal, así como la variedad y en ocasiones la ambigüedad de las posibles interpretaciones de esta experiencia”. p. 15.

Tomando en consideración los argumentos expuestos, es por lo que una tercera conducta que se trabajó fue la identificación del yo, como un repertorio de entrada para el resto del aprendizaje.

\section{Diseño de la intervención}

Se utilizó el enfoque de investigación de sujeto único, denominado también caso único o N:1 (Hersen y Balorw (1976), Kazdin (1978, 1980). Se trabajaron los diseños de investigación intra series en donde se "pretende poner de relieve los cambios de una serie de datos en una medida única o serie de medidas" (Mayer y Labrador, 1984) p. 131.

Se utilizó el diseño de investigación de criterio cambiante y múltiples $\mathrm{AB}$, en donde se investigaron los efectos de una condición sobre una conducta específica. (Kazdin, 1980).

El diseño de criterio cambiante fue propuesto por Hall y colaboradores en 1970, el que se basó en el proceso de moldeamiento. En este tipo de estudio se establecen de antemano los criterios para alcanzar la conducta del sujeto, y una vez que uno es alcanzado, se va aumentando el nivel de dificultad.

De acuerdo con Mayer y Labrador (1984) se consideraron tres aspectos importantes:

Se estableció una línea base para cada conducta de tal manera que se pudiera 
determinar que los cambios posteriores no fueran producto de la casualidad, sino efecto de la intervención, entendiendo por línea base, el nivel de entrada de los conocimientos previa intervención.

Se examinó la variabilidad de los datos para verificar la duración de cada fase.

Una vez adquirido el repertorio que se trabajaba, se tomó una nueva línea base, para determinar los niveles de entrada para la nueva habilidad que se pretendía enseñar, lo que permitió ir variando el criterio, hacia conductas con mayores niveles de dificultad, de tal manera que el diseño de criterio cambiante y múltiples $\mathrm{AB}$ pudiera llevarse a cabo. En donde A es la línea base y B la intervención lo que se representa gráficamente al final de este trabajo.

En el gráfico final se exponen la línea base uno y la intervención uno, línea base dos intervención dos, línea base tres, intervención tres, línea base cuatro, intervención cuatro, línea base cinco, intervención cinco, para uno de los niños, a manera de ejemplo, lo que permite visualizar el proceso.

La "causalidad en las investigaciones de sujeto único, está determinada por las distintas fases que cada diseño comprende, de tal manera que los cambios de una fase a otra y los cambios que se den en las fases siguientes, nos dan una valoración global y confiable de la efectividad de los procedimientos de intervención utilizados. (Troutman, A. 1982).

Al cambiar la conducta sucesivamente en cada uno de los niños, de acuerdo con el nivel operacional de la conducta de entrada, se demostró control experimental del proceso.

El control de la validez interna de este tipo de diseño está implícito en el establecimiento de la línea base.

Castro (1977) dice en relación con la línea base que ésta,

"resuelve automáticamente los problemas de "historia" creando una "historia reciente" bajo el control experimental, el de la maduración ya que cualquier cambio importante puede ser advertido, solo los que son producto de la manipulación experimental se repetirán bajo una de las formas de replicación y el de regresión estadística, ya que ese problema pierde su fuerza cuando hay un gran número de observaciones sobre el mismo sujeto" (p. 193).

Otro aspecto que se tomó en cuenta fue el de registro repetido de las conductas a intervenir por más de un observador, esto permitió una mayor confiabilidad de los datos.

La validez interna de este tipo de diseño se da a través del control experimental, el que se llevó a cabo por medio del manejo sistemático de las variables que se estaban manipulando de la definición operacional de las conductas y de la misma flexibilización del diseño.

Se utilizaron dos tipos de variables. La dependiente que se refiere a las conductas que se quieren cambiar como control de llanto, permanecer sentado, entre otras y la independiente que fueron los procedimientos empleados en el proceso como, reforzadores primarios, sociales moldeamiento y extinción.

\section{Tipo de registro}

Se llevaron registros de observación que consisten en ir anotando lo que va sucediendo en un momento dado. Dentro de esta técnica se eligió el de eventos, definido por Hall (1977) como aquel en donde un observador hace un registro acumulativo de eventos discretos de cierta clase, que produce un resultado numérico.

\section{Condicionamiento}

Se realizó un muestreo de reforzadores para determinar la efectividad de los mismos en cada niño. Esto se llevó a cabo con la ayuda de los padres, y mediante prueba y error.

Como parte de los reforzadores primarios se utilizaron galletas, cereal y 
yogurt, y como reforzadores sociales, el decirle a los niños iqué bien que estás sentado! hasta encadenar el permanecer sentado y el establecimiento del yo con las órdenes dadas, que eran: siéntese o véame.

En los períodos iniciales los horarios de reforzamiento fueron continuos cada respuesta buena se daba un reforzador. Entendiendo por reforzador como aquel estímulo contingente a un comportamiento que incrementa o mantiene la fuerza (tasa, duración) del mismo. Sulzer y Mayer (1990).

\section{Definición operacional de las conductas}

Permanecer sentado

Se definió esta conducta como la habilidad del niño de permanecer en su silla de trabajo, con la espalda apoyada al respaldar, y las manos sobre la mesa por un período mayor de 40 minutos.

\section{Contacto ocular}

Habilidad del niño para mover sus ojos de tal manera que se fijaran en los ojos de otra persona que estaba frente a él, cada vez que se le hablaba o se le daba la orden de "véame".

\section{Control del llanto}

Gritos emitidos por el niño lo suficientemente altos que se oían por lo menos a una distancia de 10-15 metros por un período mayor de 30 segundos.

\section{Identificación del yo}

Habilidad del niño para responder en forma oral con el pronombre "yo" y dirigiendo su mano hacia su cuerpo ante la pregunta ¿quién es? (en este momento se decía el nombre del niño).

\section{Resultados}

A continuación se hace un análisis estadístico descriptivo de los datos obtenidos durante las sesiones de línea base e intervención. Como puede observarse en la tabla 1, los porcentajes de entrada obtenidos por cada uno de los sujetos son muy bajos. Las sesiones uno y dos representan la línea base, y a partir de la tercera sesión se introducen los procedimientos de intervención, en donde empiezan a notarse los cambios, los que se visualizan a través de los porcentajes de respuesta alcanzados.

Tabla 1

Conducta permanecer sentado

Tiempo en minutos

\begin{tabular}{ccccc}
\hline & $\begin{array}{c}\text { No. } \\
\text { sesión }\end{array}$ & $\begin{array}{c}\text { Sujeto } \\
\text { No. } 1\end{array}$ & $\begin{array}{c}\text { Sujeto } \\
\text { No. } 2\end{array}$ & $\begin{array}{c}\text { Sujeto } \\
\text { No. 3 }\end{array}$ \\
\hline Línea base & 1 & $15 \mathrm{~min}$ & $4 \mathrm{~min}$ & $30 \mathrm{~min}$ \\
Intervención & 2 & $10 \mathrm{~min}$ & $3 \mathrm{~min}$ & $30 \mathrm{~min}$ \\
& 3 & $20 \mathrm{~min}$ & $7 \mathrm{~min}$ & $60 \mathrm{~min}$ \\
& 4 & $30 \mathrm{~min}$ & $8 \mathrm{~min}$ & $60 \mathrm{~min}$ \\
& 5 & $60 \mathrm{~min}$ & $14 \mathrm{~min}$ & $60 \mathrm{~min}$ \\
& 6 & $60 \mathrm{~min}$ & $28 \mathrm{~min}$ & \\
& 7 & $60 \mathrm{~min}$ & $60 \mathrm{~min}$ & \\
& 9 & $60 \mathrm{~min}$ & $60 \mathrm{~min}$ & \\
& 10 & & $60 \mathrm{~min}$ & \\
& 11 & & $60 \mathrm{~min}$ & \\
\end{tabular}

\section{Gráfico 1}

Permanecer sentado

Tiempo en

minutos

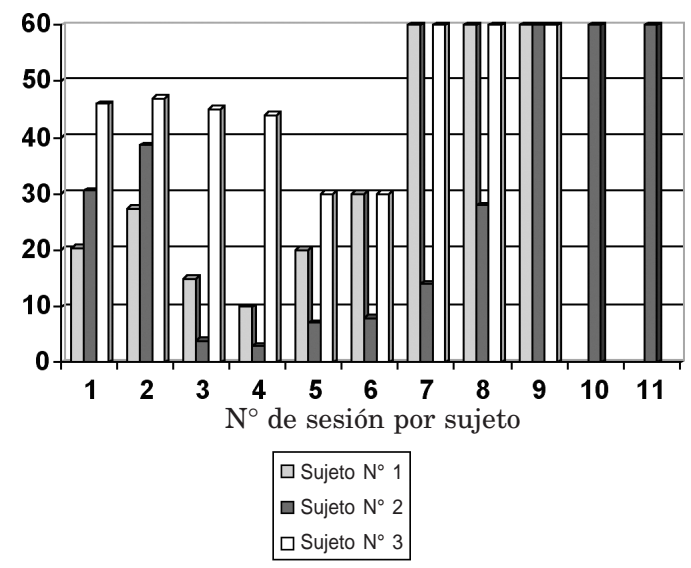


En la línea horizontal se representa el número de sesiones y en la vertical el tiempo de permanecer sentado de cada niño en minutos. La línea base para los tres niños se representa en las sesiones 1, 2 y 3 . Para el sujeto numero 1 se utiliza el color azul, para el sujeto numero 2 el color rojo y para el sujeto numero 3 el color amarillo. Puede observarse que los niveles de rendimiento para esta conducta son muy bajos, tiempo que se fue incrementando una vez que se introdujeron los procedimientos conductuales, alcanzando niveles de 60 minutos para cada uno de los niños. El éxito en esta conducta fue muy importante, ya que preparó a los niños para situaciones de aprendizaje posteriores, aumentó los niveles de concentración y fue generalizada a otros ambientes como el hogar, en donde podían permanecer sentados en la mesa por períodos mayores, o en situaciones en las que debían realizar alguna tarea específica.

Tabla 2

Conducta concepto de yo

\begin{tabular}{lrrrr}
\hline & $\begin{array}{c}\text { No. } \\
\text { sesión }\end{array}$ & $\begin{array}{c}\text { Sujeto } \\
\text { No. 1 }\end{array}$ & $\begin{array}{c}\text { Sujeto } \\
\text { No. 2 }\end{array}$ & $\begin{array}{r}\text { Sujeto } \\
\text { No. 3 }\end{array}$ \\
\hline Línea base & 1 & $0 \%$ & $0 \%$ & $35 \%$ \\
Intervención & 2 & $0 \%$ & $0 \%$ & $40 \%$ \\
& 3 & $0 \%$ & $10 \%$ & $50 \%$ \\
& 4 & $0 \%$ & $44 \%$ & $40 \%$ \\
& 5 & $5 \%$ & $93 \%$ & $65 \%$ \\
6 & $0 \%$ & $40 \%$ & $100 \%$ \\
7 & $0 \%$ & $65 \%$ & $100 \%$ \\
& 8 & $7 \%$ & $29 \%$ & $100 \%$ \\
9 & $30 \%$ & $60 \%$ & $100 \%$ \\
& 10 & $56 \%$ & $30 \%$ & \\
11 & $53 \%$ & $49 \%$ & \\
12 & $60 \%$ & $90 \%$ & \\
13 & $50 \%$ & $80 \%$ & \\
14 & $75 \%$ & $90 \%$ & \\
15 & $80 \%$ & $95 \%$ & \\
16 & $75 \%$ & $95 \%$ & \\
17 & $80 \%$ & $100 \%$ & \\
18 & $80 \%$ & $100 \%$ & \\
19 & $85 \%$ & $100 \%$ & \\
\hline
\end{tabular}

\section{Gráfico 2}

Concepto de "yo"

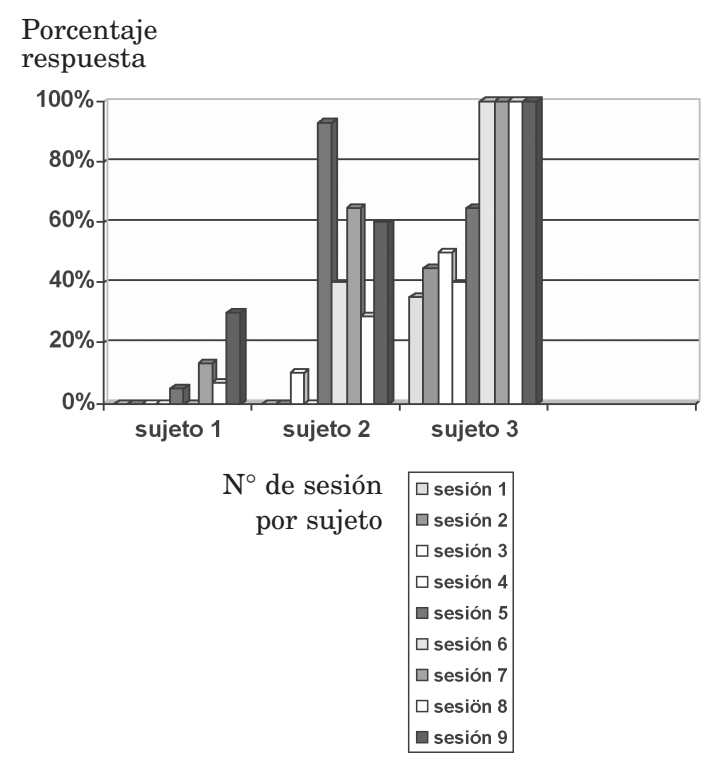

\section{Comentario}

En el gráfico se agrupa el número de sesiones por sujeto de tal manera que pueda visualizarse el avance de cada uno de los niños.

La identificación del yo, la toma de conciencia, son elementos importantes que le permiten al niño una mejor adaptación al mundo que le rodea. Antes de la intervención, el promedio de la línea base fue de $11,5 \%$ en la respuesta, y al final de ella, los tres niños incorporaron en su repertorio conductual este concepto. Los tiempos menores en la respuesta para los sujetos No. 2 y No. 3, pudieron deberse a la efectividad del reforzador primario, no así para el No. 1 para quien no fue posible determinar en la fase de muestreo de reforzadores, uno que fuera efectivo, más bien rechazaba todo comestible. En el gráfico se representan las primeras 9 sesiones, que fue el tiempo promedio de adquisición de la conducta en dos de los niños. El efecto de un buen reforzador, puede notarse en la rapidez de la respuesta, al seleccionarse 
reforzadores efectivos, lo que puede apreciarse en los sujetos No. 2 y No. 3. Como se muestra en la tabla, el sujeto No. 1 duró 19 sesiones.

\section{Conducta intermedia}

En esta área se trabajaron aquellas habilidades que permitieran a los niños responder a las diferentes órdenes, las que se sostienen sobre la base de la anterior. En esta área se trabajaron comportamientos más complejos como el lenguaje receptivo.

Es importante su desarrollo, para permitirle al niño dar respuestas sin necesidad de usar la comunicación oral. Se anotan algunas de ellas como:

- $\quad$ Seguimiento de instrucciones: deme, tome, ponga delante, ponga detrás entre otros.

- Discriminación de objetos: vaso, cuchara, tenedor, zapatos entre otros.

- Identificación y funcionalidad de partes del cuerpo: cabeza, ojos, manos, nariz, entre otros.

Para una mejor efectividad en la respuesta, cada conducta se dividió en sus componentes lo que se hizo por medio del análisis de tareas. Se siguió el proceso de encadenamiento, que se ha definido como "aquel reforzamiento de combinaciones de conductas sencillas que ya figuran en el repertorio del individuo, para formar conductas más complejas" (Sultzer-Azaroff y Mayer, 1990).

\section{Intervención}

De la misma forma que en la fase anterior, se procedió a determinar la línea base para cada una de las conductas que se trabajaron. Se utilizó instigación física que se ha definido como "un estímulo discriminativo auxiliar presentado a fin de ocasionar una respuesta dada." Sultzer y Mayer. P. 657. En los períodos iniciales, para establecer la conducta, la instigación fue total, de tal forma que se procedía a tomar la mano del niño y dirigirla hacia el objeto, que se le pedía como respuesta a las órdenes, y luego, fue parcial hasta eliminar el contacto físico con el niño.

Se trabajó la identificación de objetos a través de la orden deme, los conceptos espaciales como delante, detrás sobre debajo, así como la identificación de partes del cuerpo y colores.

Para efectos de este artículo se eligieron las conductas trabajadas: cuchara, mío y color verde, como ejemplos representativos. Es importante tomar en consideración que estos repertorios, tuvieron a su vez otras conductas de entrada que no se están presentando en este informe por razones de espacio.

Tabla 3

Discriminación y concepto de cuchara

\begin{tabular}{ccccc}
\hline & $\begin{array}{c}\mathrm{N}^{\circ} \\
\text { sesión }\end{array}$ & $\begin{array}{c}\text { Sujeto } \\
\text { No. 1 }\end{array}$ & $\begin{array}{c}\text { Sujeto } \\
\text { No. 2 }\end{array}$ & $\begin{array}{r}\text { Sujeto } \\
\text { No. 3 }\end{array}$ \\
\hline Línea base & 1 & $0 \%$ & $0 \%$ & $0 \%$ \\
& 2 & $40 \%$ & $80 \%$ & $40 \%$ \\
intervención & 3 & $50 \%$ & $75 \%$ & $50 \%$ \\
& 4 & $30 \%$ & $64 \%$ & $30 \%$ \\
& 5 & $50 \%$ & $90 \%$ & $50 \%$ \\
& 6 & $60 \%$ & $80 \%$ & $60 \%$ \\
& 7 & $90 \%$ & $100 \%$ & $70 \%$ \\
& 8 & $80 \%$ & $100 \%$ & $80 \%$ \\
& 9 & $90 \%$ & $100 \%$ & $90 \%$ \\
\hline
\end{tabular}

Gráfico 3

Conducta: Discriminación y concepto de cuchara

Porcentaje de respuesta

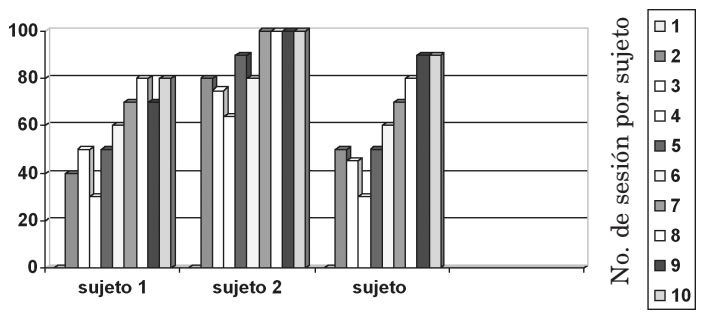




\section{Comentario}

Los datos obtenidos, están representados en términos porcentuales. La conducta de identificar objetos como cuchara, se realizó con mayor facilidad, al haber incorporado los niños repertorios como el prestar atención, por períodos de tiempo suficientes que permitieran trabajar otras habilidades, además de permanecer sentados, y seguir algunas instrucciones.

Tabla 4

Situaciones de pertenencia concepto "mío"

\begin{tabular}{lrrrr}
\hline & $\begin{array}{c}\text { No. } \\
\text { sesión }\end{array}$ & $\begin{array}{r}\text { Sujeto } \\
\text { No. 1 }\end{array}$ & $\begin{array}{r}\text { Sujeto } \\
\text { No. 2 }\end{array}$ & $\begin{array}{r}\text { Sujeto } \\
\text { No. 3 }\end{array}$ \\
\hline Línea base & 1 & $0 \%$ & $50 \%$ & $0 \%$ \\
Intervención & 2 & $0 \%$ & $60 \%$ & $0 \%$ \\
& 3 & $30 \%$ & $100 \%$ & $40 \%$ \\
& 4 & $20 \%$ & $100 \%$ & $30 \%$ \\
& 5 & $40 \%$ & $100 \%$ & $50 \%$ \\
& 6 & $50 \%$ & $100 \%$ & $60 \%$ \\
& 7 & $60 \%$ & & $70 \%$ \\
& 8 & $70 \%$ & & $90 \%$ \\
& 9 & $90 \%$ & & $80 \%$ \\
& 10 & $90 \%$ & & $90 \%$ \\
& 11 & & & $90 \%$ \\
\hline
\end{tabular}

Gráfico 4

Concepto mío

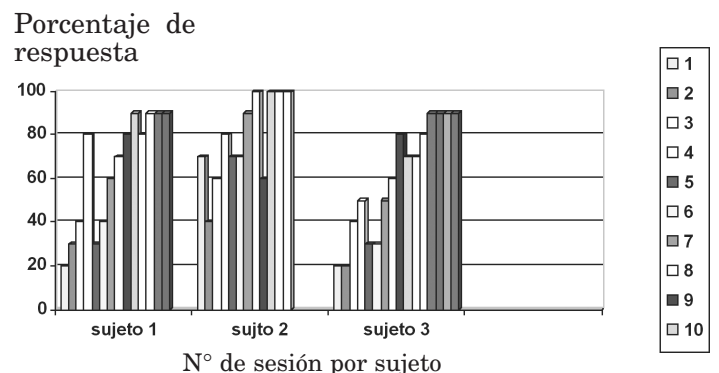

nos dé una idea de la intervención de inicio a fin, con resultados iniciales y finales.

Hay una línea base inicial, la que se nombra como Línea base uno, sobre la que estructura el aprendizaje, y una intervención uno, la que los lleva a la conducta final deseada para esa línea base.

El permanecer sentado, se convierte en requisito de entrada, para trabajar el concepto de "yo". Se establece una segunda línea base para esta conducta la que se denomina línea base 2 y una intervención 2.

Una vez alcanzados niveles de respuesta adecuados para esta conducta, se establece una tercera línea base, para la 
conducta "respuesta a órdenes", que necesita de requisitos de entrada, el que el niño permanezca sentado y se reconozca como persona, y así sucesivamente.

\section{Cuadro 6}

Conductas trabajadas sujeto No. 1, línea base intervención

\begin{tabular}{lccccc}
\hline Conducta $\rightarrow$ & $\begin{array}{c}\text { Perm. sentado } \\
\text { Línea base. } \\
1\end{array}$ & $\begin{array}{c}\text { Concepto } \\
\text { yo } \\
\text { Línea base 2 }\end{array}$ & $\begin{array}{c}\text { Concepto } \\
\text { Cuchara } \\
\text { Línea base } 3\end{array}$ & $\begin{array}{c}\text { Situa. perte- } \\
\text { nencia } \\
\text { Línea base 4 }\end{array}$ & $\begin{array}{c}\text { Conoc. } \\
\text { color } \\
\text { Línea base 5 }\end{array}$ \\
\hline Inicio Inter. $\rightarrow$ & 15 minutos & $0 \%$ & $0 \%$ & $0 \%$ & $20 \%$ \\
Final Inter. $\rightarrow$ & 60 minutos & $85 \%$ & $90 \%$ & $90 \%$ & $90 \%$ \\
\hline
\end{tabular}

Gráfico 6

Diseño de criterio cambiante

Porcentaje de respuesta

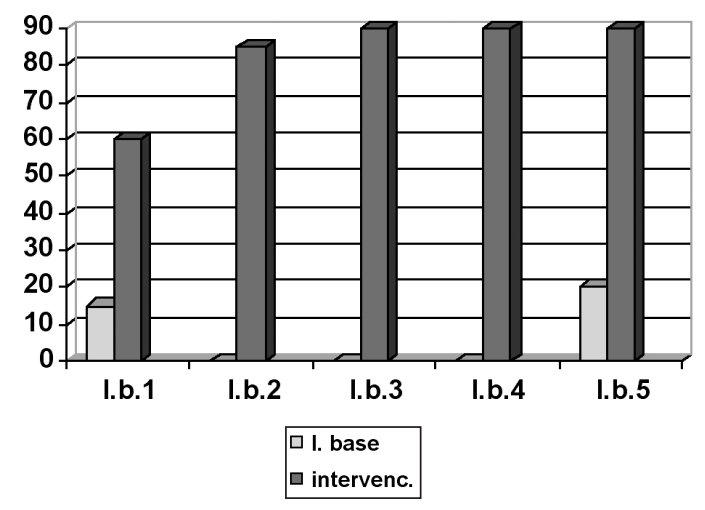

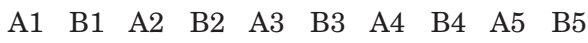
Línea base (A) intervención (B)

\section{Comentario}

Para cada una de las conductas, se establecieron de antemano los criterios a alcanzar. Los niveles de rendimiento esperados para determinar si los niños habían alcanzado la conducta fueron de un $85 \%$ en su respuesta, porcentaje que se había venido trabajando, y demostrado que ellos permitían un nivel de funcionalidad adecuado. Una vez que el niño alcanzaba los criterios, los niveles de dificultad para la conducta siguiente aumentaron, de tal manera, que la conducta anterior sirvió como requisito de entrada para la siguiente, esto permitió ir moldeando la conducta hasta alcanzar la habilidad final deseada.

Se investigaron cuáles fueron los efectos de los procedimientos conductuales empleados, los que se muestran en los niveles de rendimiento finales alcanzados por cada uno de los niños.

\section{Conclusiones}

El efecto de la terapia conductual, se refleja en los resultados obtenidos por los niños a través del proceso de intervención.

Se da un condicionamiento inicial con los reforzadores primarios, los que se aplicaron en las primeras sesiones en forma continua, (cada respuesta, un reforzador). Posteriormente se pasaron a horarios de razón fija tres, seis, ocho, hasta horarios de reforzamiento intermitente, los que en una última fase se sustituyeron por reforzadores sociales.

La escogencia de reforzadores primarios efectivos, garantizaron la adquisición de las conductas. Para ellos fue necesario el tener más de uno que fuera efectivo, sobre todo al introducir una habilidad nueva, debido a que el uso constante de uno solo perdía su efectividad. Una vez adquiridos niveles apropiados en sus respuestas, es necesario hacer el cambio hacia reforzadores más naturales, para facilitar el regreso a rutinas normales. 
El uso de registros sistemáticos de eventos, con el apoyo de otros observadores, permitieron llevar a cabo un análisis confiable y graduado del avance de cada uno de los niños.

Como puede observarse en los cuadros y gráficos, los tres niños obtuvieron las conductas propuestas una vez finalizada la intervención educativa, lo que demuestra la eficacia del tratamiento.

$\mathrm{Al}$ aumentar los niños los niveles de tolerancia a la frustración, disminuyeron las conductas autodestructivas, además de que se desarrolló una mejor relación con sus padres y personas que les rodeaban.

El ordenar las conductas es un factor importante en la implementación de programas conductuales educativos, ya que unos repertorios sirven como base para establecer sobre ellos comportamientos cada vez más complejos.

Otro procedimiento conductual eficaz en el proceso fue el "moldeamiento", en el que cada conducta se dividió en sus componentes, y se reforzó cada aproximación en forma sucesiva a la conducta final deseada.

Cada campo de habilidades que adquiere el niño se convierte a su vez en básicos para la adquisición de nuevas conductas, y en requisitos de entrada para el resto del aprendizaje, entendiendo como campo de habilidades aquellas conductas similares entre sí, y cuya diferencia entre una y otra es muy pequeña, por lo que la dificultad entre sí es muy poca. El ordenar las conductas permite una mejor sistematización de los programas.

Los programas de los niños con autismo se sustentan en la repetición constante, para facilitar el aprendizaje y que el niño asimile con mayor facilidad las habilidades que se pretenden enseñar.

Dentro de este tipo de programas, la selección de los reforzadores se convierte en un factor importante. Estos, ayudan notablemente en el proceso de aprendizaje de los niños con autismo.

Un programa conductual bien aplicado, permite desarrollar las habilidades indispensables y corroborar a través de análisis sistemáticos de los datos, el efecto de los mismos en la adquisición de las diferentes habilidades en los niños.

\section{Referencias bibliográficas}

Alberto P. Troutman, A. Applied behavior analysis to teachers, Ohio: Charles E. Merrill, 1982.

American Psychiatric Association (2000): Diagnostic and Statistical Manual of Mental Disorders. (4 Edition-texto revisado) (DSM-IV-TR). Washington D.C.. American Psychiatric Association. 1998.

Cabezas H. "Desarrollo de un programa de lectura con un niño con autismo, utilizando terapia conductual aplicada”. En: Memoria del segundo Congreso de las Américas sobre lecto escritura, Heredia, Costa Rica, EUNA, 1996.

Cabezas, H. "Uso del condicionamiento operante para la adquisición del contacto ocular en cuatro niños autistas." Revista Educación 16, 19-23. San José, Costa Rica. 1992.

Cabezas, H. "El niño con autismo: un programa estructurado para su educación": en Caballo, V. y Simón M. Manual de Psicología clínica infantil y del adolescente. Ediciones Pirámide, Grupo Anaya. España 321-345, 2000.

Coleman, M. y Gillberg, Ch. El autismo Bases Biológicas. Barcelona, Martínez Roca, 1989.

Ley 7600 Igualdad de oportunidades para las personas con discapacidad. En el Diario Oficial La Gaceta $\mathrm{N}^{\circ}$ 102, 29. San José Costa Rica, 1996. 
Hall, R. y Fox R. An alternarive applied Behavior Análisis procedure, (en Mayor y Labrador, Manual de Modificación de Conducta Editorial Alambra, Madrid, 1984.

Hersen, M. y Barlow, D. Single-case experimental desingns: Strategies for studying change, New York, Pergamon Press, 1976.

Hobson, R. P.: El autismo y el Desarrollo de la Mente. Madrid, Alianza, 1995.

Kanner, L. "Problems of nosology and Psychodynamics of early infantile Autism." American Journal o Orthopsychiatry, 1949, 19 416-426.

Kazdin, A. Statistical análisis for single case experimental designs en Mayor y Labrador, pp. 125, 1984.

Kerlinger, F. Investigación del Comportamiento. Técnicas y Metodología. México: Interamericana. 1985.

Martos, J.; Riviere A. Compiladores (autismo). Comprensión y explicación actual del autismo. Secretaría general de Asuntos Sociales. Asociación de padres de niños autistas. Imprime Artegraf, 2001.

Mayor J., y Labrador F. Manual de Modificación de conducta. Editorial Alambra, Madrid, 1984.

Vayer, P. El niño frente al mundo. Editorial Científico Médica, México, 1977.

Riviere A.; Martos J. (compiladores) El tratamiento del autismo. Nuevas perspectivas. Ministerio de Trabajo y Asuntos sociales. Imprime Artegraf. Madrid España, 1998.

Steven, R. Forness, E. y Kenneth, A. Autistic Children in School: The role of the Pediatricaln. Pediatric Annal, 1984, 13 (4) 3129328.

Sulzer B. Mayer R. Procedimientos del Análisis Conductual Aplicado a niños y Jóvenes. México: Trillas, 1990.

Hannia Cabezas Pizarro

Escuela de Orientación y Educación Especial Universidad de Costa Rica 